chung und interpartikulärer Beanspruchung durch die abrupte Stauchung des Pfropfens vor der Prallplatte zerstört werden.

Zerkleinerungsergebnisse aus Versuchen mit Glaskugeln werden hier präsentiert. Die farbliche Markierung definierter Bereiche des Partikelpfropfens ermöglicht eine detaillierte, ortsaufgelöste Untersuchung der Zerkleinerungseffizienz. Umlagerungen innerhalb des Pfropfens während seiner pneumatischen Beschleunigung in einem vertikalen Schussrohr werden durch geeignete konstruktive Maßnahmen verhindert, sodass der Aufbau des Pfropfens beim Verlassen des Schussrohrs seinem ursprünglichen Aufbau vor der Beschleunigung entspricht. Auf diese Weise sind definierte Startbedingungen für die DEM-Simulationen realisiert. Die Einflüsse verschiedener Betriebsparameter wie Ausgangspartikelgröße, Aufprallgeschwindigkeit und Pfropfenhöhe auf das Zerkleinerungsergebnis werden diskutiert.

Einzelkorn-Prallexperimente dienen zur Bestimmung der Parameter eines von Vogel [1] vorgestellten Zerkleinerungsmodells. Anhand der Ergebnisse der Einzelkornexperimente wird das verwendete DEM-Modell hinsichtlich der maximal auftretenden Druckkräfte während eines Kontakts kalibriert. Die Beschreibung der Wechselwirkungen zwischen den Partikeln bzw. einem Partikel und der Prallplatte erfolgt mittels des Kontaktmodells nach Hertz Mindlin. Zwischen den Ergebnissen aus DEMSimulationen und Experimenten ergeben sich gute Übereinstimmungen.

[1] L. Vogel, W. Peukert, Chem. Eng. Sci. 2005, 60, 5164.

\title{
P5.14
}

\section{Co-Kristallisation - Ein Verfahren zur Produktgestaltung energetischer Materialien}

\author{
U. Förter-Barth ${ }^{1)}$, Dr. M. Herrmann ${ }^{1)}$ (E-Mail: michael.herrmann@ict.fraunhofer.de), Dr. P. B. Kempa ${ }^{1)}$, Prof. U. Teipel $^{2)}$ \\ 1) Fraunhofer ICT, J.-V.-Fraunhofer-Straße 7, D-76327 Pfinztal \\ 2) Fachhochschule Nürnberg, Mechanische Verfahrenstechnik, Wassertortstraße 10, D-90489 Nürnberg \\ DOI: $10.1002 /$ cite. 200750325
}

Co-Kristalle repräsentieren neue Strukturmodelle, die insbesondere in der Pharmaindustrie großes Interesse erfahren, da anstelle einer additiven Kombination der Eigenschaften der Komponenten eine überproportionale Wirkungssteigerung im Co-Kristall erzielt werden kann. Der Begriff Co-Kristall wird für ganz unterschiedliche Strukturen verwendet. Im einfachsten Fall werden Co-Kristalle als Produkte einer Co-Kristallisation von zwei oder mehreren Komponenten definiert. Konkreter sind strukturell orientierte Definitionsversuche, die die Wechselwirkung der Komponenten im Produkt beschreiben, z. B. nicht-kovalente Bindungen.
Co-Kristallisationsversuche wurden mit den energetischen Materialien Tetramethylentetranitramin (HMX) und Trimethylentrinitramin (RDX) begonnen. Ein erster Versuch bestand darin, die Oberfläche je eines HMX- und eines RDX-Partikels anzulösen und die Partikel in Kontakt zu bringen. Nach Verdampfung des Lösemittels haften die beiden Partikel fest aneinander. Weitere Versuche umfassten die Verdampfungskristallisation, die Fällung und die Kühlkristallisation von Lösungen und Lösungsgemischen, auch unter Verwendung von Impfkristallen. Die Verfahren lieferten ein breites Probenspektrum, das von sehr feinen Pulvern bis zu grobkörnigen Partikeln reicht.

Zur Analyse der hergestellten Partikel wurden die Lichtmikroskopie, das Rasterelektronenmikroskop und die Röntgenpulverdiffraktometrie eingesetzt. Die abbildenden Methoden liefern insbesondere bei den grob-körnigen Partikeln Informationen über Partikelaufbau und Habitus. Sie eignen sich zum Nachweis grober Co-Kristalle, geben jedoch kaum Informationen über den Bindungsstatus. Eine Unterscheidung der Komponenten auf Basis der Kristallstruktur liefert die Röntgenpulverdiffraktometrie.

\section{P5.15}

\section{Ein neuartiger Magnetfilter zur selektiven Bioseparation}

Dipl.-Ing. C. Eichholz ${ }^{1)}$ (E-Mail: christian.eichholz@mvm.uka.de), Dipl.-Ing. M. Stolarski ${ }^{1)}$, Dipl.-Ing. M. Silvestre ${ }^{2)}$, PD Dr.-Ing. M. Franzreb ${ }^{2)}$, Prof. Dr.-Ing. H. Nirschl ${ }^{1)}$

1) Institut für Mechanische Verfahrenstechnik und Mechanik, Universität Karlsruhe (TH), Straße am Forum 8, D-76131 Karlsruhe ${ }^{2)}$ Institut für Technische Chemie, Forschungszentrum Karlsruhe, Hermann-von-Helmholtz-Platz 1 D-76344 Eggenstein-Leopoldshafen DOI: 10.1002/cite.200750090

Die Biotechnologie wird als die Schlüsseltechnologie für das 21. Jahrhundert bezeichnet. Allerdings bündelt die Aufbereitung des Zielprodukts in der Biotechnologie im Downstream Processing heute teilweise bis zu $80 \%$ der Betriebs- und Investitionskosten. Im Sinn einer kostengünstigen und nachhaltigen Biotechnologie wird daher vermehrt die Aufmerksamkeit auf die Entwicklung und Anwendung neuer hybrider Trenntechniken gelegt. Ein Beispiel hierfür ist die selektive Bioseparation. Dabei werden spezielle Magnetbeads auf Polymerbasis mit eingebetteten magnetischen Nanopartikeln und einer auf das Zielprodukt abgestimmten Oberflächenfunktionalisierung als Trägerpartikel 
eingesetzt. Die Abtrennung des Zielprodukts erfolgt dann aufgrund der Eigenschaften des magnetischen Trägermaterials.

Eine mögliche Umsetzung des Verfahrens ist die magnetfeldüberlagerte Kuchenfiltration. Die Laborapparatur besteht aus einer großvolumigen Drucknutsche, eingelassen in die Bohrung eines Elektromagneten. Ein integrierter Rührer hilft bei der Redispergierung der Magnetbeads für folgende Prozessschritte.

Durch das überlagerte Magnetfeld ist es möglich, den Aufbau des Filterkuchens zu verhindern oder den eines poröseren Filterkuchens $\mathrm{zu}$ forcieren. Dadurch kann nach der Adsorption des Zielprodukts an den Trägerpartikeln zunächst die mit Ionen, Zellbruchstücken u. A. verunreinigte Suspension nahezu ungehindert abfließen. Bei anschließender Waschung und Elution kann durch die Kombination von Verdünnungs-

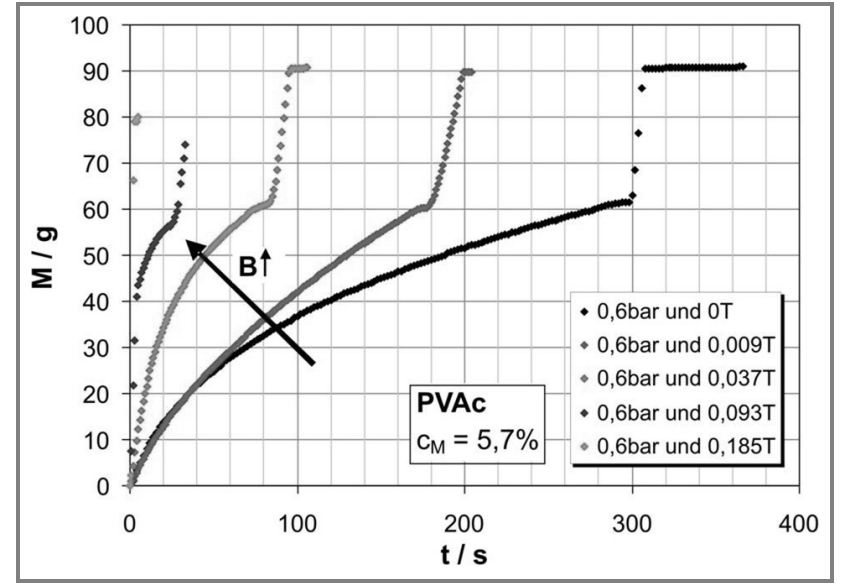

Abbildung. Filtratmassekurven für verschiedene magnetische Feldstärken: Bei 0,185 T wird der Filterkuchenaufbau vollständig verhindert.

und Verdrängungswäsche ein hoher Aufreinigungsgrad erzielt werden.

Der Vorteil dieses Verfahrens liegt in der Reduzierung der Anzahl der Aufbereitungsschritte und dementsprechend
Investitionsaufwand, Energieaufwand und Produktverlust bzw. in der Steigerung von Ausbeute und Reinheit der gewonnenen Produkte.

\title{
P5.16
}

\section{Hochfrequente Permeatrückspülung zum Einsatz bei der Mikro- und Ultrafiltration}

\author{
Dipl.-Ing. C. N. Koh ${ }^{1)}$ (E-Mail: koh@ivt.rwth-aachen.de), Prof. Dr.-Ing. T. Melin ${ }^{1)}$ \\ ${ }^{1)}$ Institut für Verfahrenstechnik, RWTH-Aachen, Turmstraße 46, D-52056 Aachen \\ DOI: $10.1002 /$ cite.200750022
}

Mikro- und Ultrafiltration liegen das Prinzip eines porösen Filters zugrunde, d. h. Teilchen, die größer sind als die Porenweite der Membran, werden vollständig zurückgehalten und lagern sich auf der Membran ab. Die so entstehende Deckschicht (engl. Fouling) bildet demzufolge einen zeitlich anwachsenden Strömungswiderstand, der zu einer Absenkung des Permeatflusses führt.

Eine oft angewandte Prozessführung zum Abtrag der Foulingschicht ist der Cross-Flow-Betrieb. Mit der Überströmung werden Scher- und Auftriebskräfte an der Oberfläche erzeugt und abgelagerte Partikel können aus der Deckschicht in der Kernströmung zurückgeführt werden. Diese Technik ist jedoch wegen des hohen Energieverbrauchs für schnelle Überströmung einerseits und der geringen Effektivität bei niedrigen Geschwindigkeiten und der dann häufig auftretenden Kanalverblockung in den Modulen andererseits nur begrenzt wirksam.
Eine weitere Methode zum Deckschichtabtrag ist die Permeatrückspülung. Für ein kurzes Zeitintervall wird durch eine permeatseitige Druckerhöhung eine Flussumkehr durch die

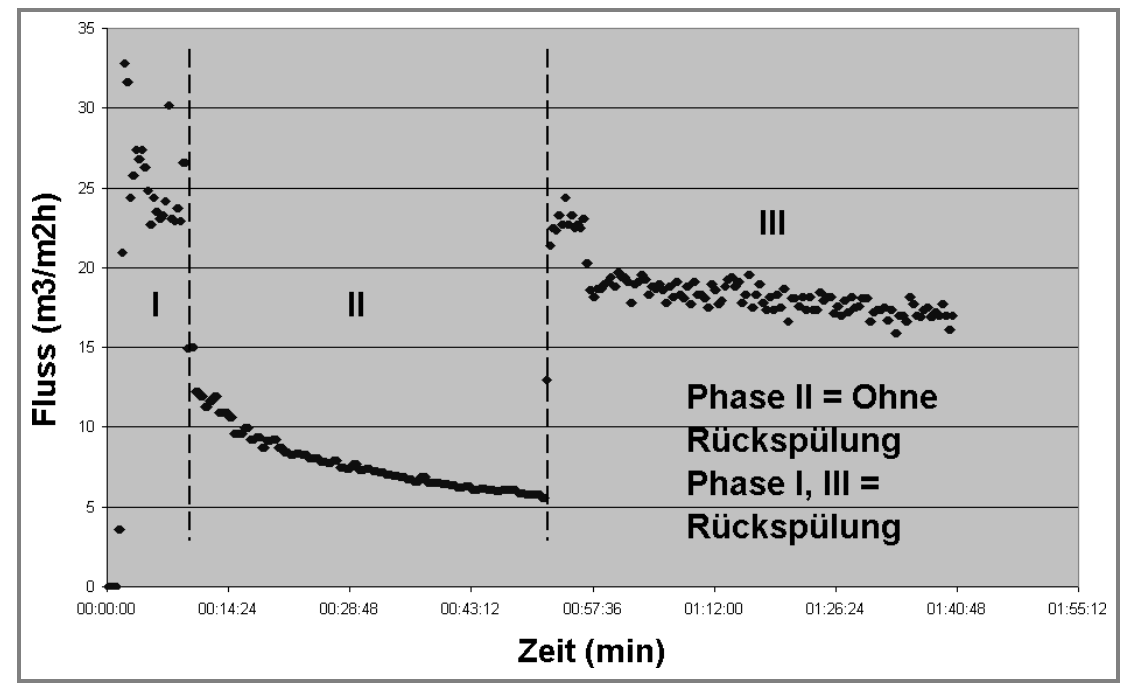

Abbildung. Einfluss der hochfrequenten Rückspülung auf Permeatfluss für Latexsuspension. 